\title{
Vortex avalanches at one thousandth the superconducting transition temperature
}

\author{
R. J. Zieve, T. F. Rosenbaum, and H. M. Jaeger \\ The James Franck Institute and Department of Physics, The University of Chicago, Chicago, Illinois 60637 \\ G. T. Seidler \\ NEC Research Institute, 4 Independence Way, Princeton, New Jersey 08540
}

G. W. Crabtree and U. Welp

Materials Science Division, Argonne National Laboratory, Argonne, Illinois 60439

(Received 28 August 1995; revised manuscript received 31 October 1995)

\begin{abstract}
We study the nonequilibrium dynamics of vortex motion at millikelvin temperatures in untwinned single crystals of $\mathrm{YBa}_{2} \mathrm{Cu}_{3} \mathrm{O}_{7-\delta}$. Above threshold fields of many tesla, flux jumps appear in the magnetic hysteresis $B(H)$. These jumps correspond to a change of 750 vortices, on average, under our micrometer-sized Bi Hall probes. Not only are the threshold fields large, but they, and the characteristics of the flux jumps themselves, are essentially independent of magnet ramp rate or sample thickness, militating against a thermally triggered instability. Moreover, the threshold differs significantly on the ascending and descending branches of the hysteresis loop. We argue for a dynamical origin for these vortex avalanches and invoke a sandpile analogy to guide our detailed explorations of the $H-T$ plane. [S0163-1829(96)02818-4]
\end{abstract}

\section{INTRODUCTION}

The thermodynamically stable state for a type-II superconductor in a magnetic field is an arrangement of vortices with uniform density, so that the macroscopic magnetic field is constant throughout the sample. In general, this state is not reached. As an external field is applied, vortices enter the sample at its edges and move towards its center. Eventually, a vortex density gradient corresponding to the critical current $J_{c}$ is established throughout the sample. ${ }^{1}$ If the external field is stabilized, then the internal field profile relaxes gradually towards the thermodynamic equilibrium. Without the influx of vortices maintaining a steep density gradient, however, the vortex motion slows dramatically due to pinning, and equilibrium is not reached on any practical time scale.

In this paper, we discuss the nonequilibrium dynamics of vortex motion along this flux density gradient, deep in the superconducting state. As the external field is changed at some fixed ramp rate, a steady state is built up in which the local magnetic flux gradient is maintained by vortex pinning forces. Whenever this balance is disturbed, the vortex arrangement becomes unstable and motion occurs. Vortices can flow continuously and in a smooth fashion or, at low driving rates, intermittently as a series of abrupt avalanches. During macroscopic, "catastrophic"' flux jumps, thousands of vortices throughout the sample participate and possibly even drive the sample temporarily into the normal state., Small, "noncatastrophic" avalanches also have been observed in the penetration of flux through tubular samples. ${ }^{4,5}$

A main issue that has emerged in the literature concerns the nature of the instability leading to vortex avalanches. In particular, there is the question to what extent the avalanches are triggered by magnetothermal instabilities, global or local, ${ }^{6}$ and sustained by thermal runaway of at least part of the sample. Catastrophic avalanche behavior due to thermal runaway, within this picture, is expected to exhibit one pre- dominant length scale and, hence, a narrow avalanche size distribution.

A different approach to vortex avalanches is based on the observation that similar instabilities characterize many other dissipative dynamical systems. In such systems, the external driving force naturally results in a steady state that not only is far from equilibrium, but right at the border to unstable behavior. Examples include electric breakdown in semiconductors, avalanching down the slope of sandpiles, and earthquakes. A recent, and very general, model for this behavior has been the concept of self-organized critically (SOC). ${ }^{7}$ Within SOC, the transition into the avalanching state would be second order and characterized by a power-law distribution of event sizes. Very wide distributions, compatible with power laws over some range and possibly indicative of collective vortex motion on many length scales, have been found in the recent experiments by Field et al. ${ }^{5}$ On the other hand, experiments on different systems in this class, such as real sandpiles, ${ }^{8}$ have shown that there are self-organized steady states that need not be critical states and, instead, display hysteresis as well as narrow size distributions.

We present here a study of a new type of macroscopic flux jump, one not triggered by thermal instability. ${ }^{9}$ We have measured the local magnetization of very clean, untwinned $\mathrm{YBa}_{2} \mathrm{Cu}_{3} \mathrm{O}_{7-\delta}$ single crystals at temperatures below $1 \mathrm{~K}$, where the quantum tunneling of vortices becomes significant. ${ }^{10}$ The magnetic field was applied perpendicular to the $\mathrm{CuO}_{2}$ planes. Our results differ from previously observed avalanche behavior in several significant ways: The avalanches exist only above well-defined threshold fields of several tesla in magnitude, as opposed to the low-field limit; the avalanche onset is essentially independent of the rate at which the external field changes, a distinct contrast to flux jumps originating in a thermal instability; there exist different threshold fields on the ascending and descending branches of the hysteresis loop, indicating an unprecedented 


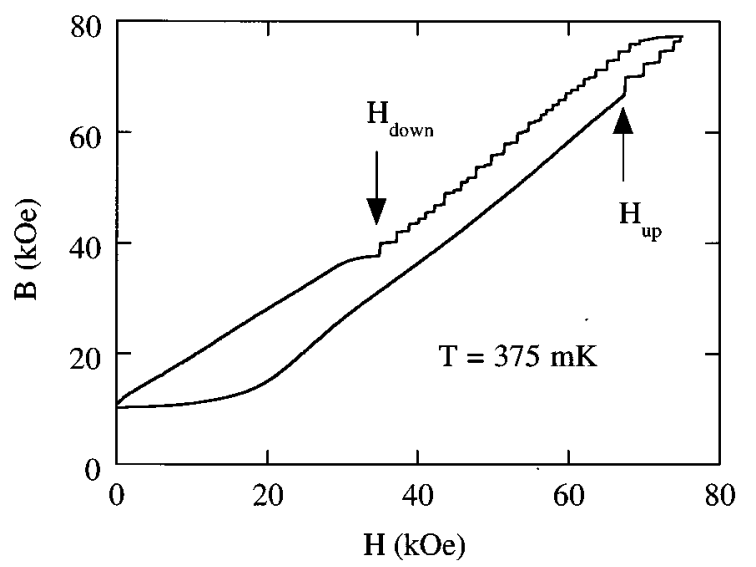

FIG. 1. Hysteresis curve of external field $H$ vs internal field $B$ measured locally with a $\mathrm{Bi}$ Hall probe on an untwinned single crystal of $\mathrm{YBa}_{2} \mathrm{Cu}_{3} \mathrm{O}_{7-\delta}$. The threshold fields for vortex avalanches differ on the ascending, $H_{\text {up }}$, and descending, $H_{\text {down }}$, branches of the loop.

level of sensitivity to magnetic field history; and the event size distribution is strongly peaked with a characteristic size, clearly distinct from the broad power laws in the SOC concept.

We describe the experimental technique and present the data in Sec. II. Specifically, we delineate the occurrence of avalanching as a function of applied magnetic field $H$ and temperature $T$. By taking a variety of paths through the $H-T$ plane, we demonstrate the dynamical origin of the avalanches. In Sec. III, we review some of the key features associated with thermally triggered vortex jumps and argue that the behavior observed in our samples is different. Many of the central aspects of vortex dynamics can be visualized easily by comparison with the motion of grains down the slope of a sandpile, a connection stressed by de Gennes. ${ }^{11}$ We use this analogy to speculate about the nature of the observed instability and to discuss our results in light of theories based on SOC. Section IV contains a brief summary and conclusions.

\section{EXPERIMENTAL RESULTS}

The samples of single-crystal $\mathrm{YBa}_{2} \mathrm{Cu}_{3} \mathrm{O}_{7-\delta}$ were grown by a conventional flux method in a gold crucible and were either untwinned as grown or detwinned by applying uniaxial stress at elevated temperature. ${ }^{12}$ The crystals had superconducting transition temperatures $T_{c}$ of $92 \mathrm{~K}$, with a narrow inductive width $\Delta T_{c}<0.2 \mathrm{~K}$. Overall, four separate crystals were investigated, one of which was later broken into three pieces to permit a study of volume effects. The measurements of the local, internal magnetic field $B$ were made with photolithographically prepared Bi Hall probes ${ }^{9}$ with typical active areas $3 \mu \mathrm{m} \times 5 \mu \mathrm{m}$. The high- $T_{c}$ crystals were mounted on the Hall probes using a thin layer of vacuum grease and were oriented with the $c$ axis along the applied magnetic field $H$.

We plot in Fig. 1 a typical hysteresis loop. These data were taken at $T=0.375 \mathrm{~K}$ on a crystal with dimensions (1.05 $\times 0.5 \times 0.05) \mathrm{mm}^{3}$, where the Hall probe was positioned close to the center. At any point on the sample, we see a loop

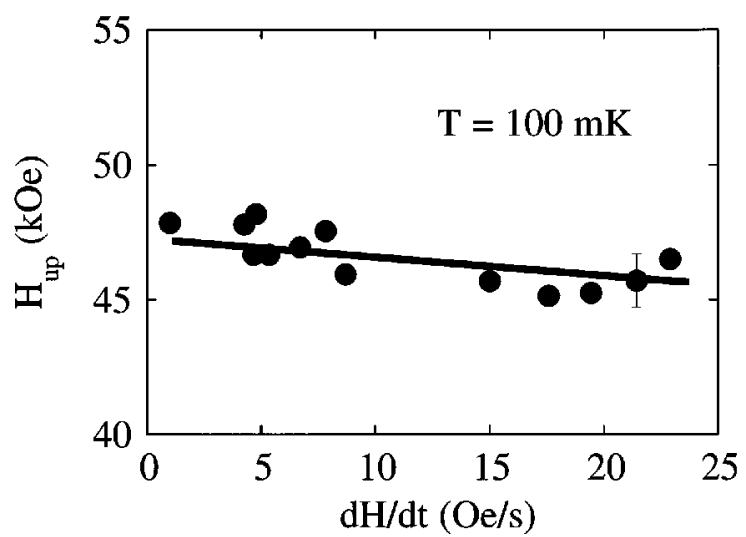

FIG. 2. Variation of the threshold field $H_{\text {up }}$ with magnet ramp rate at our lowest temperature, where heating effects should be most profound. The line is a guide to the eye.

similar to that in Fig. 1. Upon ramping up the external field $H$, the internal field $B$ changes from a continuous dependence on the applied field to a series of abrupt steps. These steps indicate a sudden change in the internal magnetic flux due to vortex avalanching. An average-sized step in this figure corresponds to a change in flux of 750 vortices beneath the probe. The first occurrence of avalanching happens at many tens of kilooersteds and defines an onset field $H_{\text {up }}$. After our maximum field $H \approx 75 \mathrm{kOe}$ is reached and the ramp direction is reversed, the steps continue down to a lower threshold field $H_{\text {down }}$, beyond which the avalanches cease.

We show, in Fig. 2, $H_{\text {up }}$ as a function of magnet ramp rate at our lowest temperature $T=0.1 \mathrm{~K}$, where heating effects should be most pronounced. The threshold field for vortex avalanching changes by at most $4 \%$ for more than a factor of 20 change in ramp rate. This small change in $H_{\text {up }}$ barely exceeds a large step size and militates against heating from vortex motion as a major causal factor for the avalanche onset.

We turn now to a consideration of the symmetry between the two branches of the hysteresis loop, the most unusual

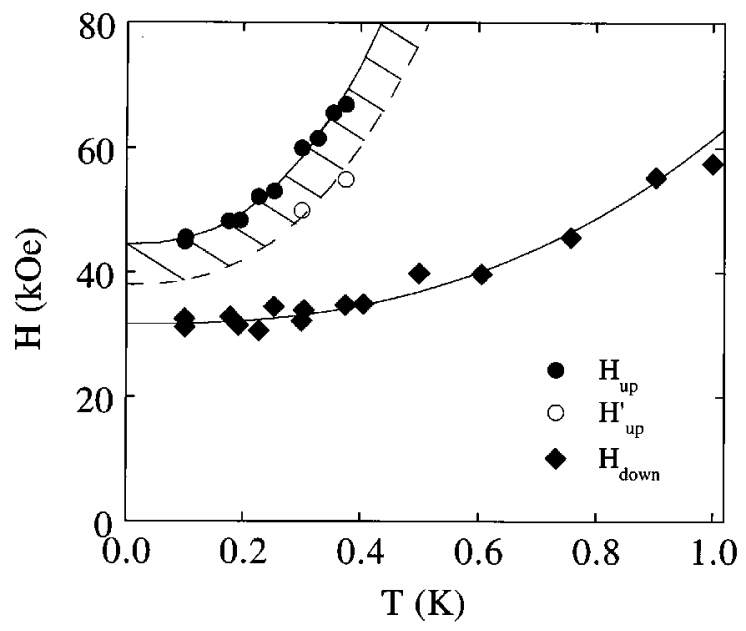

FIG. 3. Temperature dependence of the threshold fields for avalanche behavior (Fig. 1). $H_{\text {up }}$ can be shifted by up to $10 \mathrm{kOe}$ by altering the internal field profile (see text), but it never coincides with $H_{\text {down }}$. Solid lines are least-squares fits following an empirical $T^{5 / 2}$ power law. 


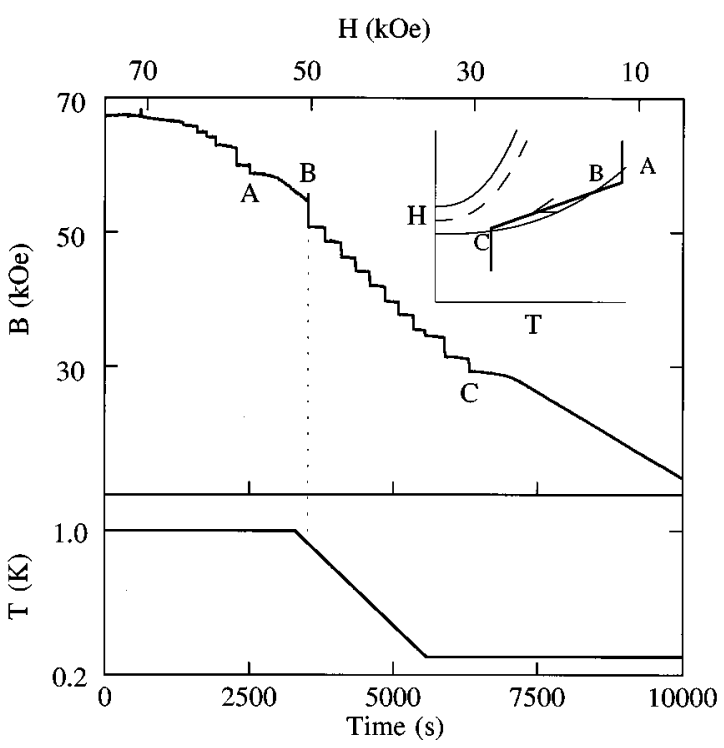

FIG. 4. Effect of changing temperature (bottom) on step formation in $B(H)$ (top). The path through the $H-T$ phase diagram of Fig. 3 is sketched in the inset. There is no evidence for the type of hysteretic behavior found at a first-order transition: Vortex avalanches begin and end where expected.

feature of our steps. We present in Fig. 3 a "phase diagram", for the magnetization jumps. The solid symbols are the locations of the first and last magnetization steps in hysteresis loops of the form shown in Fig. 1. The solid lines are fits through these data to the empirical form $H_{\text {up/down }}=H_{0}+A T^{n}$, with $n=2.5 \pm 0.2$. The $H_{\text {down }}$ line is repeatable over long times and on different cooldowns. However, the points on the $H_{\text {up }}$ curve can move by a few kOe between cooldowns or on a time scale of several weeks while cold. ${ }^{13}$ Nonetheless, a clear difference between the $H_{\text {up }}$ and $H_{\text {down }}$ curves remains, making Fig. 3 resemble a phase diagram at a first-order transition.

In order to test for hysteresis between the regimes where vortices move continuously and where they cascade in steps, we have explored various paths through the $H-T$ plane.

(1) We have examined first the effect of changing temperature. We show in Fig. 4 one such experiment. The inset sketches our path through the phase diagram of Fig. 3. We begin at $H=75 \mathrm{kOe}, T=1 \mathrm{~K}$, and ramp the applied field down steadily at a constant rate of $7 \mathrm{Oe} / \mathrm{s}$ (upper axis of main figure). Steps appear in $B(H)$. At point $\mathbf{A}=H_{\text {down }}(T=1 \mathrm{~K})$, $B(H)$ becomes smooth. We then begin to cool to $T=0.3 \mathrm{~K}$. At point $\mathbf{B}$, shortly after the cooling begins, the steps reappear. Finally, magnetization jumps disappear again at point $\mathbf{C}=H_{\text {down }}(T=0.3 \mathrm{~K})$. This procedure involves crossing the $H_{\text {down }}$ line at three separate places. The most interesting of these is the crossing at point $\mathbf{B}$. By cooling from $1 \mathrm{~K}$, we prepare the sample on the descending branch of the hysteresis loop, but with the internal field changing continuously. If $H_{\text {down }}<H_{\text {up }}$ is a sign of a first-order transition, then $B(H)$ might remain smooth even at low temperatures after our unusual preparation. We see no such evidence for "supercooling', steps in $B(H)$ begin immediately on crossing the $H_{\text {down }}$ line of Fig. 3.

(2) We have examined the corresponding thermal pathway in which temperature increases. We begin at a high field

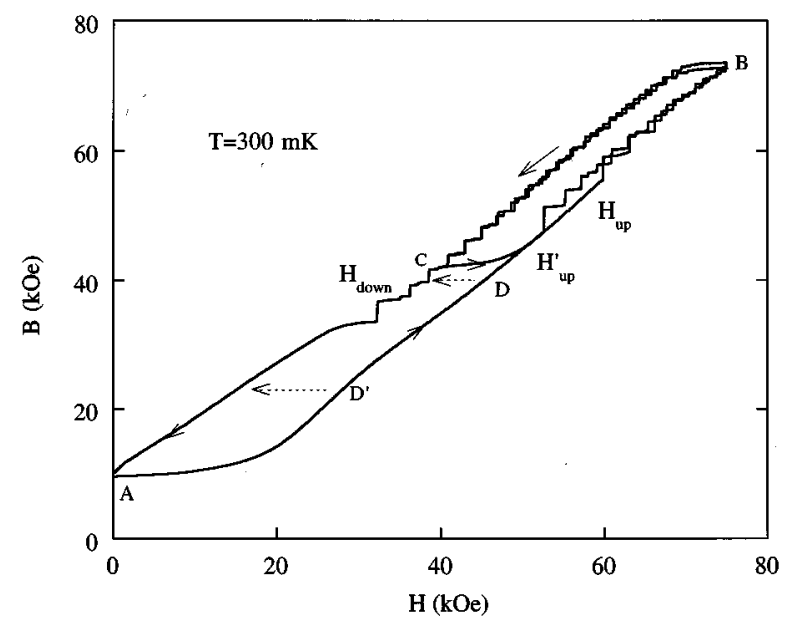

FIG. 5. Series of nested hysteresis loops. Reversing $H$ below $H_{\text {down }}$ (path $A D^{\prime} A$ ) retains smooth behavior, but reversals when $H_{\text {down }}<H<H_{\text {up }}(A D A)$ lead to avalanches on the descending branch. $H_{\text {up }}(A B)$ can be shifted down to $H_{\text {up }}^{\prime}(A B C B)$, where the maximum effect of $10 \mathrm{kOe}$ is shown, but $H_{\text {down }}$ appears insensitive to ramping sequence. Overlaid steps tend to be close, but not in lock-step.

and low temperature, and slowly decrease the magnetic field. While still in the step regime, we warm the sample. In this case, the steps cease as soon as the $H_{\text {down }}$ line is crossed at the higher temperature. The robustness of $H_{\text {down }}$ to temperature changes points to the intrinsic nature of the jump region on the downward branch.

(3) In a separate set of experiments, performed at constant temperature, we have changed the applied field sequence. Consider the full and partial hysteresis loops of Fig. 5. On originally ramping the magnetic field up from point $\mathbf{A}$ $(H=0)$ to point $\mathbf{B}(H=75 \mathrm{kOe})$, steps appeared at $H_{\text {up }} \sim 60$ kOe. The field subsequently was decreased to point $\mathbf{C}(H$ $=40 \mathrm{kOe}$ ) and then increased from $\mathbf{C}$ back to $\mathbf{B}$. Now, the steps appeared at a new, lower $H_{\text {up }}^{\prime} \sim 50 \mathrm{kOe}$, clearly demonstrating that a change in the ramping sequence can affect the appearance of steps on the increasing branch of the hysteresis loop.

By contrast, the descending branch is unaffected by the field reversal. On the path ADA, sketched in Fig. 5, the field is decreased from point $\mathbf{D}$, before any steps occur on the ramp up. Nonetheless, steps appear on the ramp down, ending at the usual $H_{\text {down }}$. Moreover, as expected, a path such as $\mathbf{A D}^{\prime} \mathbf{A}$, which crosses only between smooth regions on both branches, does not result in any avalanches. The stability of $H_{\text {down }}$ to changes in the field profile presumably is related to its repeatability on successive cooldowns.

For exploring the limits to moving $H_{\text {up }}$, the ramp sequences executed in Fig. 5 are inconvenient. The first problem is one of interpretation: Are the early magnetization jumps caused by an unusual disturbance of the field profile in the sample or by having started from the descending branch where steps already occur? The other difficulty is technical: At the lowest temperatures, the difference between $H_{\text {up }}$ and $H_{\text {down }}$ does not exceed the width of the hysteresis loop sufficiently to permit an adequate range of movement in the threshold field.

Another method of triggering steps below $H_{\text {up }}$ is more 


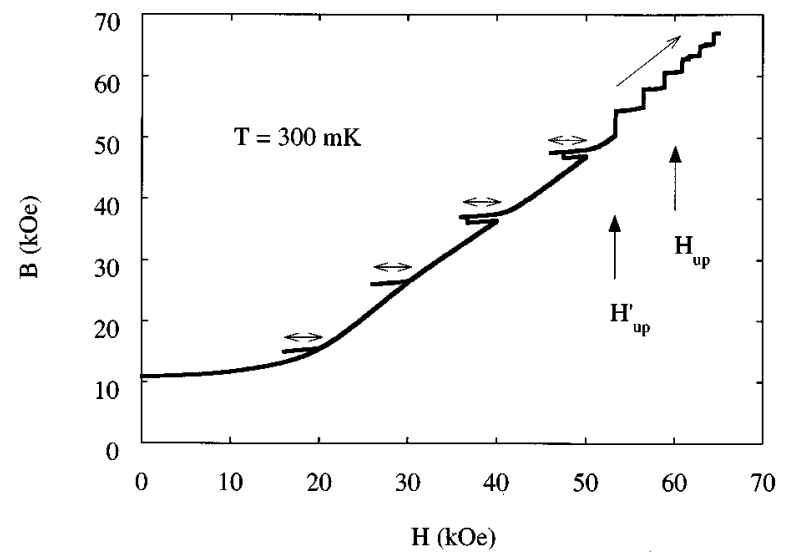

FIG. 6. Backtracks less than the width of the hysteresis loop are sufficient to decrease the threshold $H_{\text {up }}$ for vortex avalanches. Nonetheless, $H_{\text {up }}^{\prime}$ and $H_{\text {down }}$ always remain separated by more than the width of the loop; the up and down thresholds occur at different internal fields $B$.

definitive and can be used more widely. We show in Fig. 6 data on increasing $H$ from 0 to $65 \mathrm{kOe}$, with a series of 4-kOe backtracks from 20, 30, 40, and $50 \mathrm{kOe}$. Hence, the field $H$ was ramped from zero to $20 \mathrm{kOe}$, back to $16 \mathrm{kOe}$, up to $30 \mathrm{kOe}$, back to $26 \mathrm{kOe}$, and so on. Only the backtracking from $50 \mathrm{kOe}$ was followed by magnetization jumps on the ramp up, although a jump during the ramp down could be discerned on the 40-kOe segment. At $T=0.3 \mathrm{~K}$, we could not induce steps on the increasing branch before $H_{\text {up }}^{\prime} \sim 50 \mathrm{kOe}$. Thus, it does not appear possible to achieve a situation where $H_{\text {up }}$ equals $H_{\text {down }}$; there is a fundamental difference between the ramp directions. The open circles in the $H$ - $T$ diagram of Fig. 3 represent the minimum fields for steps to occur on increasing field. At $T=0.5 \mathrm{~K}, H_{\text {down }}=40 \mathrm{kOe}$, but since no avalanches could be induced on the increasing branch, $H_{\text {up }}^{\prime}$ must be greater than $75 \mathrm{kOe}$. The dashed line through the open circles of Fig. 3 follows the empirical form $H_{\mathrm{up}}^{\prime}=H_{0}$ $+A T^{2.5}$, with the constraint that $H_{\text {up }}^{\prime}(0.5 \mathrm{~K})>75 \mathrm{kOe}$. The hatched region accommodates variations arising from both magnetic and thermal cycling.

The backtracking experiments contain significant information of interest: (i) Steps can occur at $H_{\text {up }}^{\prime}<H_{\text {up }}$, even when no jumps occur during the downward section of the backtracking. (ii) Excursions as small as $1 \mathrm{kOe}$ can trigger early steps. Since this is much less than the width of the hysteresis loop, it is not necessary to start from the descending branch. The dependence of $H_{\text {up }}$ on the magnetic field's recent ramping history suggests that the origin of the steps is related to the details of the field profile within the sample. (iii) A similar reversal of the field on the descending branch has no effect on $H_{\text {down }}$. In our exploration of the avalanche region, we never were able to influence the value of $H_{\text {down }}$. Although it is possible to move $H_{\text {up }}$ by up to $10 \mathrm{kOe}$, a substantial difference between $H_{\text {up }}$ and $H_{\text {down }}$ always remains.

Finally, we turn to the characteristics of the steps. We display in Fig. 7 a pair of histograms of step sizes observed during magnetic field ramps up and down. The histograms were derived from 13 hysteresis loops between $H=0$ and 75 kOe at $T=0.1 \mathrm{~K}$. Histograms composed of a subset of hys-
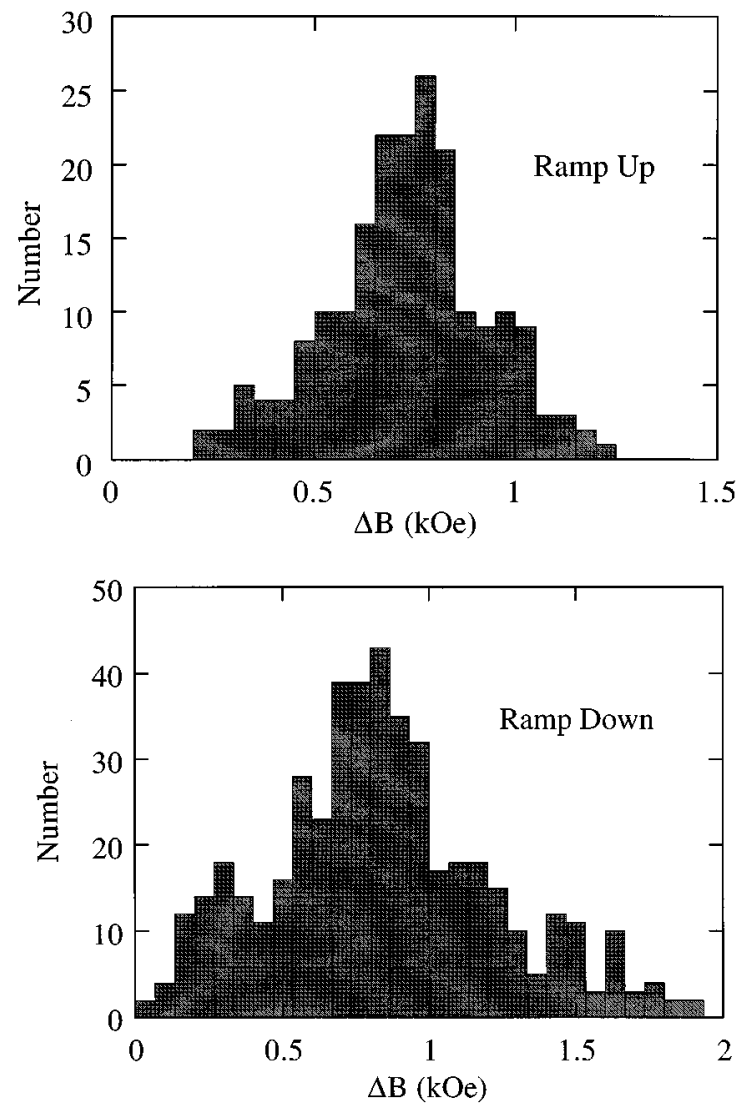

FIG. 7. Step size histograms for 13 separate hysteresis loops at $T=0.1 \mathrm{~K}$. Both the ascending (top) and the descending (bottom) branches of the hysteresis loop result in clearly peaked distributions. $\Delta B=1$ kOe corresponds to a change in density under the gaussmeter of approximately 1000 vortices.

teresis loops at the slowest and at the fastest magnet ramp rates showed no significant differences. The steps occurring on both the increasing and decreasing field branches result in sharply peaked distributions, clearly not power-law distributions, centered around average avalanche sizes of 750 flux quanta. The added breadth of the distribution on the decreasing branch of the hysteresis loop simply appears to reflect the better statistics obtained over the greater field range between $H=75 \mathrm{kOe}$ and $H_{\text {down }}$ as compared to the difference between $H_{\text {up }}$ and the maximum field.

\section{DISCUSSION}

Step structures in superconducting hysteresis loops are well known and often ensue from thermal instabilities. Near the critical current $J_{c}$, the amount of flux motion changes rapidly as depinning occurs. Above $J_{c}$, the potential energy surface for vortices is so tilted that local minima no longer appear at the pinning centers, and vortices flow rapidly through the sample. The critical current is temperature dependent, generally decreasing as $T$ rises. Thus, if the temperature rises slightly, vortices move quickly to accommodate the new, lower $J_{c}$. Vortex motion causes phase slippage and generates heat, which can further raise the temperature. A stability parameter depending on the sample's specific heat, thermal conductivity, size, and critical current describes 
when this positive feedback loop leads to thermal runaway. Very poor thermal links and/or magnet ramp rates of $\mathrm{T} / \mathrm{sec}$ are usually required to initiate the thermally activated flux jumps, and they are observed in the low-field limit.

We believe that the steps we observed in the high-field quantum regime in $\mathrm{YBa}_{2} \mathrm{Cu}_{3} \mathrm{O}_{7-\delta}$ are not triggered by thermal instabilities, despite the fact that they occur at low temperature. Given that the obvious source of heating is the motion of vortices through the sample as the field changes, one would expect the magnet ramp rate to exert a profound influence. Yet, as demonstrated in Fig. 2, the ramp rate has virtually no effect on the threshold field or on the size of the flux jumps (cf. Hsu et al. $^{3}$ ).

Our tests with samples of various sizes also argue against a thermal instability causing the jumps. All of the crystals are plates with their $c$ axes along the thin direction. They were mounted flat on the bismuth Hall probe, which made the thin dimension the important one for thermal conductivity. Nonetheless, crystals with thicknesses from 12 to $70 \mu \mathrm{m}$ behaved concordantly. The steps did depend, however, on the sample's area, becoming more likely as the area increased. As a test, we fractured a particularly large sample which exhibited flux jumps into three pieces of equal thicknesses. The smallest piece, which was approximately a rectangle with sides $0.63 \mathrm{~mm} \times 0.20 \mathrm{~mm}$, had a smooth hysteresis curve. The larger pieces - a right triangle with legs 0.88 and $0.59 \mathrm{~mm}$ and a rectangle $0.36 \mathrm{~mm} \times 0.68 \mathrm{~mm}$ - both displayed avalanche behavior, although with not as many flux jumps as the original crystal.

Introducing disorder in the form of randomly distributed columnar defects, like varying vortex length (crystal thickness), appears to have a limited influence on the avalanche behavior. Magnetization steps continued unabated in the larger crystals after irradiation with $605-\mathrm{MeV} \mathrm{Xe}$ ions. It is important to note, however, that the vortex density at $H_{\text {up }}$ or $H_{\text {down }}$ is always greater than the equivalent columnar defect density ( $\leqslant 20 \mathrm{kOe})$, so that "interstitial" vortices may dominate the response.

There are several additional, important differences between our data and hysteresis loops in samples with thermally triggered instabilities. Thermally triggered instabilities occur at low fields, with the hysteresis loops becoming smooth at high fields, opposite to what we observe. Furthermore, the jumps occur at a given sample magnetization, and they typically proceed until the magnetization is zero. In our case, the magnetization is largest at low fields, where no jumps occur. The steps do not reach the line $B=H$, at which the magnetization vanishes. In fact, near the onset field $H_{\text {up }}$, the typical avalanche size of 750 flux quanta means that the net rearrangement of vortices under the Hall probe is of order $5 \%$. Finally, the steps in $\mathrm{YBa}_{2} \mathrm{Cu}_{3} \mathrm{O}_{7-\delta}$ occur at significantly different fields on the increasing and decreasing branches of the hysteresis loop. In Fig. 1, for example, $H_{\text {up }}=67 \mathrm{kOe}$, while the field of the final jump on the decreasing branch, $H_{\text {down }}=35 \mathrm{kOe}$. This difference exists not simply because the hysteresis loop has a finite width; the steps occur for different values of the internal field $B$ (as well as the applied field $H)$. By contrast, the usual thermodynamic instability criterion $^{2}$ depends only on properties, such as the specific heat and the thermal conductivity, which are single-valued functions of $B$.
The striking differences with flux jumps of thermal origin lead us to the conclusion that the onset of avalanches in $\mathrm{YBa}_{2} \mathrm{Cu}_{3} \mathrm{O}_{7-\delta}$ crystals at millikelvin temperatures must be driven by a dynamic instability. A simple, physical picture corresponding to the magnetic flux profile inside the sample is a sandpile inside a box, ${ }^{11,14}$ a classic dynamical system. In this analog, the magnetic field $B$ corresponds to the local sand-filling level and individual flux bundles, the vortices, to single grains of sand. The vortex density gradient (or $J_{c}$ ) is given by the slope of the sandpile's free surface. Changes in the external field $H$ correspond to adding, or removing, grains at the walls of the box. In the steady state, the sandpile slope is maintained by friction between neighboring grains, just as $J_{c}$ is a result of local vortex pinning.

Avalanching in sandpiles has attracted much recent interest as a model for phase transitions (from the stable to the moving state) in highly dissipative, nonequilibrium systems. ${ }^{8}$ In sufficiently large, real sandpiles, avalanches typically build up to form system-spanning events that transfer large amounts of grains downhill and reset the slope everywhere to a new, slightly lower value than before the event. This large avalanche size occurs even in the presence of strong dissipation because of the momentum of individual sand grains once the avalanche has started. As a consequence, the grain motion is underdamped and the transition between the stable and moving states appears hysteretic and first-order-like. The result is a strongly peaked event size distribution, dominated by large avalanches. The hysteresis can be reduced and the size distribution can be widened in smaller piles (samples), where the influence of momentum buildup is decreased. Introducing vibrations (finite temperature) or increasing the ramp rate also reduces the observed hysteresis, but in this case by diminishing the effective friction (pinning) forces.

The existence of threshold fields such as $H_{\text {up }}$ or $H_{\text {down }}$ indicates that not only the field gradient, but also the field magnitude is important. In fact, both the vortex velocity and the relative strength of the local pinning forces are reduced with increasing $B$; this favors the occurrence of instabilities at high fields. Since the local vortex density increases with increasing $B$, the avalanche events we observe above $H_{\text {up }}$ proceed in a regime of densely packed, interacting flux bundles, where any motion will likely produce large-scale, plastic rearrangements. In the low-field regime, by contrast, local variations in the density gradient (i.e., in the slope) can be accommodated by elastic rearrangements of the vortex configuration. The elastic energies are smooth and have no peaks and valleys which would prevent continuous compression to accommodate a larger applied field.

During buildup of the sandpile, grains added at the walls are added at the highest locations with respect to the overall, $\mathrm{V}$-shaped surface profile (Bean state). Friction at these locations supports grains locally, but does not support much of the overall pile weight. During a steady-state ramp down, on the other hand, grains removed at the walls are removed at the lowest positions of the overall, inverted-V profile. These grains support much of the whole surface layer, and it is conceivable that, for the same total pile mass, their removal more easily triggers avalanches than the addition of grains near the top. Translating between gravity-induced and magnetic stresses, this analogy may explain the hysteresis we observe in the fields $H_{\text {up }}$ and $H_{\text {down }}$ (and in the corresponding 
internal fields $B$ ). The same argument also makes plausible the observation of steps along only the descending branch in loop ADA in Fig. 5. Furthermore, it provides insight into why $H_{\text {up }}$ varies with ramping history, while $H_{\text {down }}$ remains unchanged (Figs. 3, 5, and 6): Bringing about avalanching by adding grains to the top of the pile is much more sensitive to the exact process of addition than inducing avalanching by pulling out "keystones" at the bottom.

The sandpile analogy also has been invoked in models of self-organized criticality. In this picture, there is no characteristic length scale and the observed pattern of avalanche sizes is expected to follow a power-law distribution. Such power laws were found in experiments ${ }^{5}$ on vortex motion in niobium tubes near $T_{c}$. In the case of our $\mathrm{YBa}_{2} \mathrm{Cu}_{3} \mathrm{O}_{7-\delta}$ crystals at $0.001 T_{c}$, the step size distribution has a clear maximum (Fig. 7). As distinct from the details of when the steps occur and how they are triggered, these peaked histograms are qualitatively similar to previously observed thermally controlled magnetization jumps. When thermal runaway occurs, the flux jumps have a characteristic size related to a cutoff temperature where heating from vortex motion can be dissipated successfully.

While the onset field for vortex avalanches appears dynamical in nature, it certainly is possible that once vortices flow, thermal runaway is then the appropriate description. Alternatively, it is possible to stretch the sandpile analogy and speculate about a complete dynamical description, taking into account the extremely low temperatures of our experiments. In the case of very-low-mass vortices, the situation at first sight is different from that of finite-mass sand grains and completely overdamped motion could be expected. By analogy with sandpiles, this should lead to wide size distributions and the demise of hysteresis. At low temperatures, however, vortex tunneling becomes important, leading to mass renormalization. ${ }^{15}$ Depending on the degree of dissipation, the resulting effective mass then can be due primarily to renormalization effects, and a transition of firstorder character may be recovered.

\section{CONCLUSIONS}

We find that vortex avalanches in untwinned single crystals of $\mathrm{YBa}_{2} \mathrm{Cu}_{3} \mathrm{O}_{7-\delta}$ in the high-field quantum limit differ in key respects both with traditional, thermally generated flux jumps in type-II superconductors and with recent highresolution experiments on vortex motion through $\mathrm{Nb}$ tubes. The most striking aspect of our results is the pronounced difference between the threshold magnetic fields for avalanches on the ascending and descending branches of the hysteresis loop. This observation has motivated comparisons to dynamical instabilities in sandpiles. The exact roles played by the disorder in the vortex glass, the strong pinning, and the tunneling of vortices at millikelvin temperatures remain to be clarified.

\section{ACKNOWLEDGMENTS}

We are grateful to K. M. Beauchamp and S. N. Coppersmith for enlightening discussions. The work at the University of Chicago and at Argonne National Laboratory was supported by the National Science Foundation (DMR9120000) through the Science and Technology Center for Superconductivity. R.J.Z. acknowledges support from the MRSEC Program of the National Science Foundation under Grant No. DMR-9400379. G.W.C. acknowledges support from the U.S. Department of Energy, Basic Energy Sciences-Materials Science, under Contract No. W-31-109ENG-38.
${ }^{1}$ C. P. Bean, Rev. Mod. Phys. 36, 31 (1964).

${ }^{2}$ For a review, see Y. B. Kim and M. J. Stephen, in Superconductivity, edited by R. D. Parks (Dekker, New York, 1969), Vol. 2, p. 1107.

${ }^{3}$ For traditional flux jumps in high- $T_{c}$ superconductors, see, for example, M. Guillot et al., Phys. Lett. A 127, 363 (1988); S. W. Hsu, K. Chen, and W. H. Lee, Solid State Commun. 75, 799 (1990); A. Gerber et al., Phys. Rev. B 47, 6047 (1993); K.-H. Muller and C. Andrikidis, ibid. 49, 1294 (1994).

${ }^{4}$ C. Heiden and G. I. Rochlin, Phys. Rev. Lett. 21, 691 (1968).

${ }^{5}$ S. Field, J. Witt, F. Nori, and X. Ling, Phys. Rev. Lett. 74, 1206 (1995).

${ }^{6}$ V. A. Shklovskij, J. Low Temp. Phys. 20, 662 (1994).

${ }^{7}$ P. Bak, C. Tang, and K. Wiesenfeld, Phys. Rev. Lett. 59, 381 (1987); C. Tang and P. Bak, ibid. 60, 2347 (1988).

${ }^{8}$ For a review, see H. M. Jaeger and S. R. Nagel, Science 255, 1523 (1992).

${ }^{9}$ G. T. Seidler et al., Phys. Rev. Lett. 70, 2814 (1993).
${ }^{10}$ G. T. Seidler et al., Phys. Rev. Lett. 74, 1442 (1995), and references therein; W. Hai-hu, H. G. Schnack, R. Griessen, B. Dam, and J. H. Rector, Physica C 241, 353 (1995); A. V. Mitin, Zh. Eksp. Teor. Fiz. 93, 590 (1987) [Sov. Phys. JETP 66, 335 (1987)].

${ }^{11}$ P. G. de Gennes, Superconductivity of Metals and Alloys (Benjamin, New York, 1966).

${ }^{12}$ U. Welp et al., Physica (Amsterdam) 161C, 1 (1989).

${ }^{13}$ We originally reported (Ref. 9) that the magnetization relaxation vanished 4-6 kOe prior to the appearance of flux jumps. This observation was incorrect, a result of the sensitivity of $H_{\text {up }}$ to external factors on the few kOe scale. We now believe that the absence of measurable flux creep is a consequence, rather than a cause, of the steps in the magnetization.

${ }^{14}$ A. M. Campbell and J. E. Evetts, Adv. Phys. 21, 199 (1972); J. Souletie, J. Phys. (Paris) 44, 1095 (1983).

${ }^{15}$ G. Blatter, V. B. Geshkenbein, and V. M. Vinokur, Phys. Rev. Lett. 66, 3297 (1991). 